\title{
Time of flight for photon in human skin
}

\author{
Antra Dzerve*, Inesa Ferulova, Alexey Lihachev, and Janis Spigulis \\ Institute of Atomic Physics and Spectroscopy, University of Latvia, 19 Rainis blvd., Riga, Latvia \\ *e-mail: dzerveaa@gmail.com
}

\begin{abstract}
The time of flight for photons in human skin was measured using picosecond diode laser. Two different wavelength lasers were used $-405 \mathrm{~nm}$ and $510 \mathrm{~nm}$. A difference for time of flight in normal skin and in nevus was observed as well as a difference for different wavelength laser irradiation was observed. For $405 \mathrm{~nm}$ laser irradiation the difference was 41 ps while comparison of time of flights skin and nevi using $510 \mathrm{~nm}$ irradiation showed a result of 32 ps. Results allow to conclude that the time photon travels in skin might depend on the characteristics of the medium and wavelength of the irradiation. This can be related to known data for light penetration depth in human skin for different wavelengths. (C) 2016 Journal of Biomedical Photonics \& Engineering.
\end{abstract}

Keywords: absorption, light impulse, biophotonics, medical physics.

Paper \#3044 received 2016.05.18; revised manuscript received 2016.09.16; accepted for publication 2016.09.29; published online 2016.09.30. doi: 10.18287/JBPE16.02.030301

\section{References}

1. R. L. Siegel, K. D. Miller, and A. Jemal, “Cancer statistics, 2016,” A cancer journal for clinicians 66(1), 7-30 (2016).

2. J. Lesins, A. Lihache, R. Rudys, S. Bagdonas, and J. Spigulis, "Skin autofluorescence photo-bleaching and photo-memory," Proc. SPIE-OSA 8092, 80920N (2011).

3. I. Ferulova, A. Lihachev, and J. Spigulis, "Photobleaching effects on in vivo skin autofluorescence lifetime," J. Biomed. Opt. 20(5), 051031 (2015).

4. T. Svensson, E. Alerstam, D. Khoptyar, J. Johansson, S. Folestad, and S. Andersson-Engels, "Near-infrared photon time-of-flight spectroscopy of turbid materials up to $1400 \mathrm{~nm}$," Rev. Sci. Instrum. 80(6), 063105 (2009).

5. D. Khoptyar, A. A. Subash, S. Johansson, M. Saleem, A. Sparén, J. Johansson, and S. Andersson-Engels, "Broadband photon time-of-flight spectroscopy of pharmaceuticals and highly scattering plastics in the VIS and close NIR spectral ranges," Optics Express 21(18), 20941-20953 (2013).

6. J.-C. Bernengo, H. Adhoute, and D. Mougin, "Measurement of the time of flight of photons into the skin: influence of site, age and gender, correlation with other skin parameters," Skin Research and Technology 21(1), 25-34 (2014).

7. Safety of Laser Products - Part 1: Equipment Classification and Requirements IEC 60825-1 (2007).

8. R. R. Anderson, and J. A. Parrish, "The optics of human skin," Journal of Investigative Dermatology 77(1), 13-19 (1981).

\section{Introduction}

Skin is by far the largest organ for humans and it is also the one that comes in contact with many threats from outside world all the time - air pollution can clog pores and create rash or zits, too much time spent in the sun can result in sunburns, UV irradiation can be a cause of diseases. According to statistics, the number of diagnosed melanomas per year is growing [1]. Therefore optical diagnostics as a fast and relatively easy way for diagnosing skin diseases is getting more popular.

By time of flight one understands the time photon spends in skin before it is reflected back and exits the skin. When light enters the skin, part of it is reflected back from the surface, but part of it continues to penetrate deeper layers of skin. This light can be scattered, absorbed or reflected in many different directions. Time of flight for photon is affected by 
characteristics of the medium it travels in. Information about photon behaviour under different types of skin and skin formations is a key element to understanding many different effects connected to research aimed at improving and developing new ways for skin disease diagnostics and other experimental uses. Finding unusual formations not noticeable to naked eye by comparing results of the time light spends in patients skin with data for normal, healthy skin without the need to use surgical intrusion - this is only one of possible uses for database with photon times of flight in human skin and not dangerous formations such as nevi. This type of database can help revolutionise skin diagnostics alongside other similar researches on other topics, such as photobleaching [2], skin autofluorescence lifetime [3] and others.

Researches about time of flight of photons in different mediums such as turbid materials [4] and pharmaceuticals, also highly scattering plastics have already been carried out [5]. But the usage of photon time of flight spectroscopy undoubtedly has a place in medical spectroscopy field for human skin. This kind of research has already been carried out in with specific wavelength lasers. For example, a research was done using $1064 \mathrm{~nm}$ laser irradiation and the authors themselves suggest that measurements using shorter wavelengths should be carried out for more extensive conclusions [6].

Investigation for time of flight for photons in human skin was conducted using picosecond diode laser of wavelengths $405 \mathrm{~nm}$ and $510 \mathrm{~nm}$ and results were acquired, comparing the time of flight for photon in healthy human skin and in a nevi. For $405 \mathrm{~nm}$ irradiation difference in flight time was found at $41 \mathrm{ps}$ while for $510 \mathrm{~nm}$ laser the difference was found to be 32 ps.

\section{Method and equipment}

In order to determine the time spent under skin for photons of different wavelengths and compare data for normal, healthy skin and nevi, following experimental setup was created.

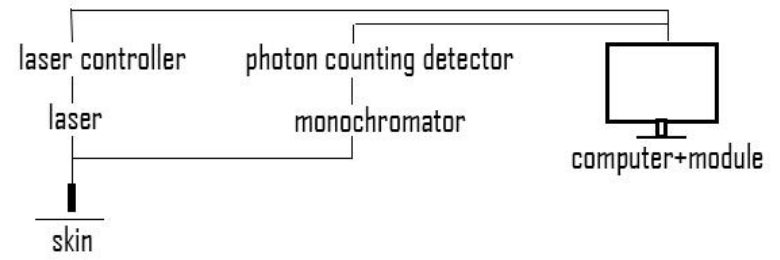

Fig. 1 Experimental setup scheme for measurements of photon time-of-flight in human skin.

The single-spot measurement setup scheme is shown in Fig. 1, it comprised a laser controller and a picosecond laser (PicoQuant: $405 \mathrm{~nm}$, pulse half-width 59 ps, repetition rate $20 \mathrm{MHz}$ mod. LDH-D-C-405, PicoQuant: $510 \mathrm{~nm}$, pulse half-width 107 ps, repetition rate $20 \mathrm{MHz}$ mod. LDH-D-C-510) with 200 micrometer silica core optical fibre output via the SMA-connector. The fibre connected to laser represented one leg of a Yshaped optical fibre bundle while other leg contained additional 6 fibres to deliver the light via a monochromator to the photon counting detector (Becker\&Hickl, PMC-100-4). Monochromator was set to allow thought the wavelength equal to the wavelength of the laser being used. This was done to eliminate possible fluorescence effects which may lead to extra emitted photons not ones that reflect inside the skin and travel through it. The laser controller and photon counting detector were connected to a PC with a data processing card (Becker\&Hickl, TCSPC, mod. SPC150). The fibre-optic probe was tightly fixed in a way that the skin surface and tip of the Y-shaped fibre bundle were in contact in such a way that skin surface and fibre create a ninety degree angle and the laser impulse goes directly into the skin straight down. That way the part of light that enters and gets reflected straight back can be observed and measured. Laser impulse was measured by using a highly reflective metal surface and measuring reflected signal to acquire the form of laser impulse. This impulse was used as a reference for comparison.

By comparing measured reflected signals to laser impulse signals from human skin visually, one can observe a shift in the peak and slight widening of the impulse. Using a time line connected to all measurements, and a signal can be properly compared to acquire peak shift in time units [6]. The error for any acquired measurement is set by time resolution of the experimental setup. For this specific case the error could be approximated as 6 ps.

Laser irradiation that was used was well below the skin laser safety limit $\left(200 \mathrm{~mW} / \mathrm{cm}^{2}\right.$, exposure time up to $103 \mathrm{~s}$ [7]. The safety and well-being of the human subjects involved in all clinical measurements was assured through the supervision of the local ethics committee that authorized the studies.

Experiments were carried out on 10 volunteers aged from 22 to 30 with skin phototype II (classified by Fitzpatrick classification). The main goal was determining if the results show different tendencies for length of photon time of flight for healthy skin and nevi.

Data were acquired from volunteers' upper back and chest regions. Differentiation between healthy skin and nevi (or distinguishing nevus from any different type of skin abnormality) was performed with the help of volunteers themselves, who had previously on their personal occasions consulted with medical personnel.

\section{Results}

A difference in time of flight for healthy skin and nevi was observed. For $510 \mathrm{~nm}$ laser a difference of 19-39 ps between time of flight in healthy skin and in nevus was observed, healthy skin having a longer time of flight. Most of the differences were around the 19 ps mark. For $405 \mathrm{~nm}$ laser a difference of 19-58 ps was observed, mostly falling in the $39-58$ ps diapason. With this 
wavelength healthy skin had longer times of flight as nevi as well. Since the times of flight were measured from comparable regions of volunteers' skin, data does not give any more description for other parts of body at the moment. More research should be conducted to compare these results to possibly different ones from other parts of body.

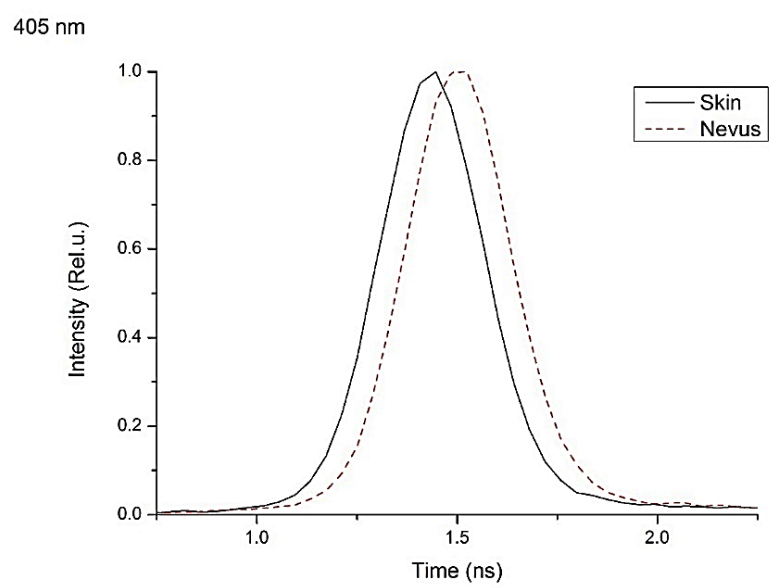

Fig. 2 Comparison of pulse from healthy skin and from nevus, using $405 \mathrm{~nm}$ laser.

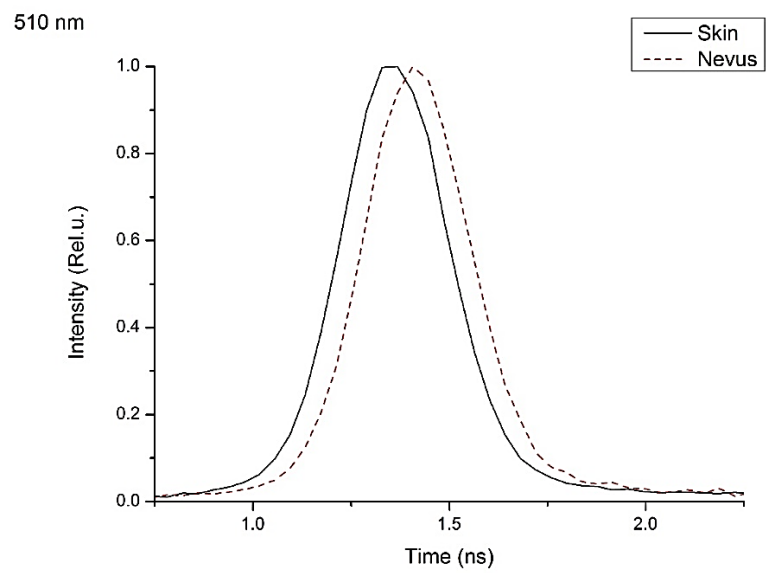

Fig. 3 Comparison of pulse from healthy skin and from nevus, using $510 \mathrm{~nm}$ laser.

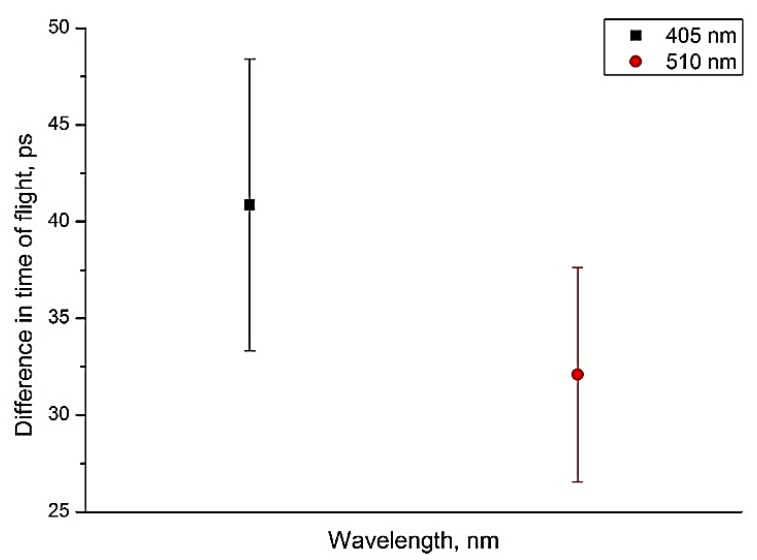

Fig. 4 Difference for time of flight in healthy skin and nevi for $405 \mathrm{~nm}$ and $510 \mathrm{~nm}$ lasers.
Examples for gathered data with $405 \mathrm{~nm}$ and $510 \mathrm{~nm}$ lasers are shown in Fig. 2 and Fig. 3. Average time of flight for both lasers is shown in Fig. 4.

Analysis of gathered data shows a tendency for length of time - times of flight for $510 \mathrm{~nm}$ laser impulses were longer than for $405 \mathrm{~nm}$ laser for healthy skin.

\section{Discussion}

As shown in previously mentioned results, it can be seen that for $510 \mathrm{~nm}$ laser times of flight are longer, compared to $405 \mathrm{~nm}$ laser irradiation times of flight. This can be attributed to penetration depth for different wavelengths of lasers. Other researches on skin and laser light interaction show this tendency with penetration depths for lasers of shorter wavelengths [8].

A constant difference in times of flight for healthy skin and nevi was observed. This is possibly connected with different characteristics of the medium the laser pulse travels in. Shorter times of flight in nevi can be attributed to higher density in tissue material. Photons that enter the skin have more points on which they can scatter - a different scattering coefficient describes nevi, compared to normal skin. This is backed up by practical measurements - those done on nevi had to have longer data acquisition times than healthy skin with no defects. Time of measurement was chosen basing on count of registered photons - it had to be the same for healthy skin and nevus to compare the data. Prolonged measurements for nevi can also be attributed to higher absorption coefficient for nevi.

This type of measurement allows to conduct diagnostics very quickly. One measurement can take from only one or two seconds up to thirty seconds, only for very specific cases it would take a minute. The shortness and specifics of one measurement is a huge advantage compared to other types of skin diagnostics no surgical intrusion, short waiting time (the patient does not have to sit still for long periods of time, thus minimising the probability of error from a simple movement), a chance for creating an interface to acquire immediate visual and numerical results.

At the moment one of limitations is time resolution. For this specific set-up, as mentioned before, the error is close to being comparable with acquired results. This, though, could be fixed or at least improved by using lasers with a more suitable frequency.

\section{Conclusions}

As shown above, the chosen experimental setup can deliver reproducible data and be used with different wavelength lasers which allows to carry out the experiment to comparably easily gather diverse results in different spectral regions.

Acquired data show a constant difference in time of flight for healthy skin and nevi. As it can be seen in acquired results, the difference in photon time of flight for $405 \mathrm{~nm}$ irradiation was found at $41 \mathrm{ps}$ while for 510 $\mathrm{nm}$ laser the difference was found to be $32 \mathrm{ps}$. 
Using this as a guideline, a database for times of flight in different types of skin can be created. The database could be used as a reference in diagnostics. Database should not be limited to only healthy skin and nevi. By adding a specialist from medical staff, differentiation between other skin formations could be made and it would allow to widen the possible database.
Further research would require a more extensive data acquisition from a larger group of volunteers using more wavelengths, optionally, in optical diapason to create a large database that could be a reference point for this type of fast, relatively easy and not time consuming diagnostics. This would not only minimise the need for surgical intervention, it could in the future be used as a standard for optical diagnostics. 